\title{
Complex intracellular calcium oscillations A theoretical exploration of possible mechanisms
}

\author{
José A.M. Borghans ${ }^{1}$, Geneviève Dupont ${ }^{*}$, Albert Goldbeter \\ Faculté des Sciences, Uninersité Libre de Bruxelles, Campus Plaine, C.P. 231, B-1050 Brussels, Belgium
}

Received 17 August 1996; revised 13 January 1997; accepted 16 January 1997

\begin{abstract}
Intracellular $\mathrm{Ca}^{2+}$ oscillations are commonly observed in a large number of cell types in response to stimulation by an extraccllular agonist. In most cell types the mechanism of regular spiking is well understood and models based on $\mathrm{Ca}^{2+}$-induced $\mathrm{Ca}^{2+}$ release (CICR) can account for many experimental observations. However, cells do not always exhibit simple $\mathrm{Ca}^{2+}$ oscillations. In response to given agonists, some cells show more complex behaviour in the form of bursting, i.e. trains of $\mathrm{Ca}^{2+}$ spikes separated by silent phases. Here we develop several theoretical models, based on physiologically plausible assumptions, that could account for complex intracellular $\mathrm{Ca}^{2+}$ oscillations. The models are all based on one- or two-pool models based on CICR. We extend these models by (i) considering the inhibition of the $\mathrm{Ca}^{2+}$-release channel on a unique intracellular store at high cytosolic $\mathrm{Ca}^{2+}$ concentrations, (ii) taking into account the $\mathrm{Ca}^{2+}$-activated degradation of inositol 1,4,5-trisphosphate $\left(\mathrm{IP}_{3}\right)$, or (iii) considering explicitly the evolution of the $\mathrm{Ca}^{2+}$ concentration in two different pools, one sensitive and the other one insensitive to $\mathrm{IP}_{3}$. Besides simple periodic oscillations, these three models can all account for more complex oscillatory behaviour in the form of bursting. Moreover, the model that takes the kinetics of $\mathrm{IP}_{3}$ into account shows chantic hehaviour.
\end{abstract}

Keywords: $\mathrm{Ca}^{2+}$ signalling; $\mathrm{Ca}^{2+}$ oscillations; Bursting; Chaos

\section{Introduction}

Intracellular $\mathrm{Ca}^{2+}$ oscillations are observed in a large variety of cell types including cardiac cells, oocytes and hepatocytes. These oscillations appear to play an important role in the control of many cellular processes. For example, secretion and egg activation at the time of fertilization are modulated by $\mathrm{Ca}^{2+}$ oscillations. The mechanism of these oscillations has

\footnotetext{
${ }^{*}$ Corresponding author.

${ }^{1}$ Present address: Theoretical Biology, Utrecht University Padualaan $8,3584 \mathrm{CH}$ Utrecht, The Netherlands.
}

been actively investigated, both from experimental (for reviews, see [1-5]) and theoretical [6-18] points of view.

Although most of the experimental data show simple periodic oscillations, some others show more complex periodic behaviour resembling bursting, in which phases of high frequency oscillations are separated by phases of quicscence, in a pattern that occurs at regular intervals. Such a complex oscillatory behaviour has been observed in pancreatic acinar cells stimulated by carbachol [19], in endothelial cells stimulated by ATP [20] and in hepatocytes stimulated by diverse chemicals, such as cyclic AMP 
[21], taurolithocholate 3-sulphate [22], diadenosine $5^{\prime}, 5^{\prime \prime \prime}-P^{1} P^{4}$-tetraphosphate [23], ATP or both ATP and cAMP [24-26]. Shown in Fig. 1 is an example of both single and complex $\mathrm{Ca}^{2+}$ oscillations observed in single (distinct) hepatocytes in response to two different stimuli.

It is now clear that the activation by cytosolic $\mathrm{Ca}^{2+}$ of $\mathrm{Ca}^{2+}$ release from internal stores plays a predominant role in generating sustained $\mathrm{Ca}^{2+}$ oscillations. This stimulatory effect has been observed for two types of $\mathrm{Ca}^{2+}$ channels located in the endoplasmic-reticulum (ER) membrane: the inositol 1,4,5-trisphosphate $\left(\mathrm{IP}_{3}\right)$ receptor $/ \mathrm{Ca}^{2+}$ channel $\left(\mathrm{IP}_{3} \mathrm{R}\right.$ channel) [27] and the ryanodine receptor $/ \mathrm{Ca}^{2+}$ channel (RyR channel) [28]. The $\mathrm{IP}_{3}$ synthesised in response to the binding of the agonist to the plasma membrane receptor leads to an increase in the cytosolic $\mathrm{Ca}^{21}$ concentration which, in turn, stimulates further release of $\mathrm{Ca}^{2+}$ from the store, leading to the generation of a $\mathrm{Ca}^{2+}$ spike. The decreasing part of the spike is generally assumed to be due to a decreased efflux from the stores originating from partial depletion of the intracellular store or/and to the inhibitory effect of cytosolic $\mathrm{Ca}^{2+}$ on the $\mathrm{IP}_{3} \mathrm{R}$ at higher $\mathrm{Ca}^{21}$ concentrations [27]. Ryanodine receptor $/ \mathrm{Ca}^{2+}$ channels play an important role in some cell types where $\mathrm{Ca}^{2+}$ pools insensitive to $\mathrm{IP}_{3}$ have been shown to be involved in the generation of $\mathrm{Ca}^{2+}$ oscillations [29].

Intracellular $\mathrm{Ca}^{2+}$ oscillations have been intensively studied in different theoretical models. In these models, oscillations are either based on $\mathrm{Ca}^{2+}$. induced $\mathrm{Ca}^{2+}$ release $(\mathrm{CICR})[6,8,12,18]$, or require (a)

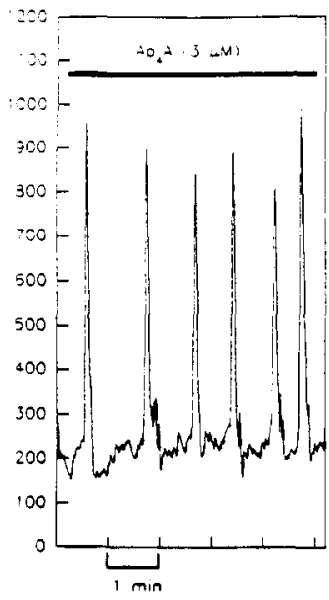

(b)

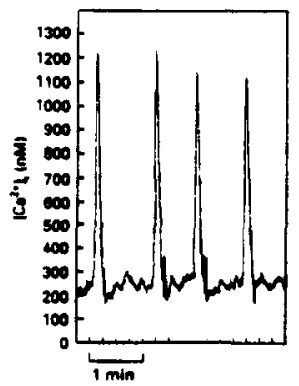

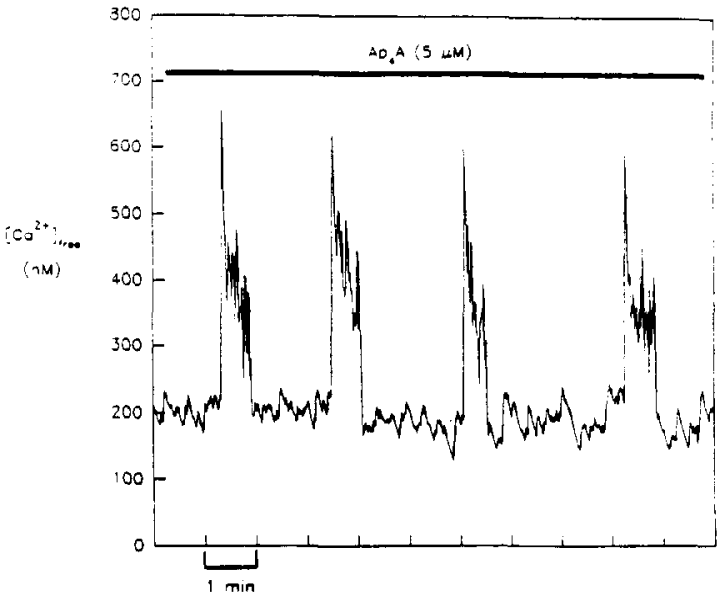

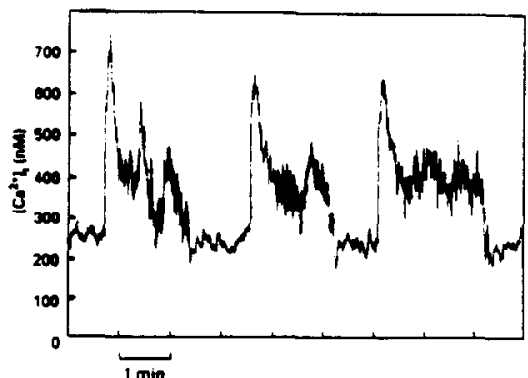

Fig. 1. Experimentally observed complex intracellular $\mathrm{Ca}^{2+}$ oscillations in single (distinct) continuously stimulated by (a) diadenosine $5^{\prime}, 5^{\prime \prime \prime}-P^{\prime} P^{4}$-tetraphosphate (3 and $5 \mu \mathrm{M}$ respectively) [23], (b) taurolithocholate 3-sulphate (300 and $400 \mu \mathrm{M}$ respectively) [22]. The mechanism for bursting proposed by the authors of these experimental studies is summarized in the text (see Section 6). (Reproduced with kind permission from the authors.) 
the periodic variation of $\mathrm{IP}_{3}[7,10]$. Some models rely on the detailed dynamics of the $\mathrm{IP}_{3}$ receptor [16,17,30,31]. However, all these models give rise only to simple periodic oscillations.

The $\mathrm{Ca}^{2+}$ signal is known to be spatially organized in many cells, where stimulation often initiates the propagation of intracellular $\mathrm{Ca}^{2+}$ waves. Thus one might think that the temporal complexity of $\mathrm{Ca}^{2+}$ signals could originate from the interplay between different $\mathrm{Ca}^{2+}$ waves within the cell. Another source of complex oscillations may reside, however, in the mechanisms underlying the generation of simple $\mathrm{Ca}^{2+}$ oscillations. Here we focus on the conditions in which complex oscillations of cytosolic $\mathrm{Ca}^{2+}$ may arise when spatial aspects are not explicitly taken into account. To this end, we extend the models based on CICR proposed by Goldbeter et al. [8] and Dupont and Goldbeter [15] in several ways. Each extended model provides another mechanism that can account for complex $\mathrm{Ca}^{2+}$ oscillations in the form of bursting. In a recent study, Shen and Larter [32] have obtained evidence for bursting and chaos in a closely related version of a model based on CICR. Coupling between CICR and a model based on the inactivation by protein kinase $\mathrm{C}$ ( $\mathrm{PKC}$ ) of the synthesis of $\mathrm{IP}_{3}$ can also give rise to $\mathrm{Ca}^{2+}$ oscillations of the bursting type [33]. Here we also present evidence for chaos and compare the occurrence of complex oscillatory behaviour in three different versions of a model based on CICR. Rather than focusing on the mechanism of complex $\mathrm{Ca}^{2+}$ oscillations in a particular cell type, our aim is to investigate the types of regulatory mechanisms that could give rise to bursting and chaos.

The first model considers both activation and inhibition of the $\mathrm{Ca}^{2+}$-release channel of a unique intracellular $\mathrm{Ca}^{2+}$ reservoir. This model is based on experimental data that show a bell-shaped dependence of the activity of $\mathrm{Ca}^{2+}$-release channels on the cytosolic $\mathrm{Ca}^{2+}$ concentration $[27,28,34,35]$. The model can account for complex oscillations of the bursting type.

The second model explicitly considers the dynamics of $\mathrm{IP}_{3}$. Relevant to the present study is the fact that inositol 1,4,5-trisphosphate 3-kinase, one of the two enzymes responsible for $\mathrm{IP}_{3}$ degradation, is significantly activated by the $\mathrm{Ca}^{2+}$-calmodulin complex [36]. Given that the calmodulin concentration is usu- ally large in all cell types and that $\mathrm{Ca}^{2+}$ binding to calmodulin is very fast, one can assume from a modelling point of view that $\mathrm{Ca}^{2+}$ directly stimulates the degradation of $\mathrm{IP}_{3}$. The coupling of this negative feedback loop to the positive CICR loop appears to be sufficient to generate bursting and chaos. This approach must be viewed as complementary to the one proposed by Shen and Larter [32]. In their model, the activation of the $\mathrm{IP}_{3}$ production by cytosolic $\mathrm{Ca}^{2+}$, reported in some cell types, is considered as the second destabilizing mechanism which generates bursting when coupled to CICR.

Bursting often occurs in a region of the parameter space in which two mechanisms capable of producing oscillations become active [37-39]. However, in a third model, based on the two-pool CICR model by Goldbeter et al. [8], we show a rather unusual kind of bursting that occurs in a model containing a single destabilizing mechanism; this oscillator is however modulated by a variable input, which is inherent in the dynamics of the system. We show indeed that the existence of two variable $\mathrm{Ca}^{2}$ stores, one sensitive and the other one insensitive to $\mathrm{IP}_{3}$, is sufficient to produce bursting oscillations.

\section{The basic one-pool model for simple periodic $\mathrm{Ca}^{2+}$ oscillations}

Because our first two models are based on the one-pool CICR model proposed by Dupont and Goldbeter [15], we will briefly recall this model here, before extending it in Sections 3 and 4. In the one-ponl CICR model two variables are considered: the concentration of cytosolic $\mathrm{Ca}^{2+}, Z$, and the concentration of $\mathrm{Ca}^{2+}$ in the intracellular pool, $Y$. The model is schematized in Fig. 2. The cytosolic $\mathrm{Ca}^{2+}$ concentration $Z$ changes due to the influx of extracellular $\mathrm{Ca}^{2+}\left(V_{\text {in }}\right)$, the passive efflux of $\mathrm{Ca}^{2+}$ from the cytosol into the extracellular medium $(k Z)$ and from the intracellular $\mathrm{Ca}^{2+}$ store into the cytosol $\left(k_{\mathrm{f}} Y\right)$. Moreover, $\mathrm{Ca}^{2+}$ is pumped into $\left(V_{2}\right)$ and released from $\left(V_{3}\right)$ the intracellular store. The model equations are given by:

$$
\begin{aligned}
& \frac{\mathrm{d} Z}{\mathrm{~d} t}=V_{\mathrm{in}}-V_{2}+V_{3}+k_{\mathrm{f}} Y-k Z, \\
& \frac{\mathrm{d} Y}{\mathrm{~d} t}=V_{2}-V_{3}-k_{f} Y,
\end{aligned}
$$




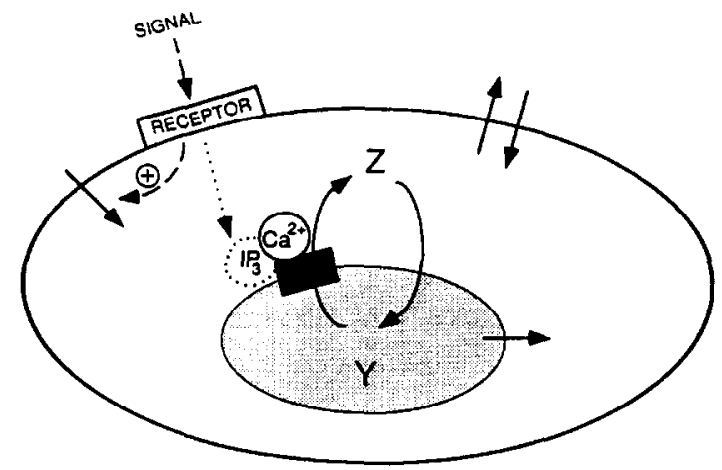

Fig. 2. Schematic representation of the one-pool model for $\mathrm{Ca}^{2+}$ oscillations based on CICR. $\mathrm{Ca}^{2+}$ fluxes are denoted by solid arrows. $Z$ and $Y$ represent cytosolic and intravesicular $\mathrm{Ca}^{2+}$. respectively. If the inhibition of the $\mathrm{Ca}^{2+}$ channel on the intracellular pool is taken into account (Eq. (3)) this model could also account for bursting. If the channel represents a RyR channel, the dotted part of the figure should not be considered (see text for details).

with:

$V_{\text {in }}=v_{0}+v_{1} \beta$,

$V_{2}=V_{\mathrm{M} 2} \frac{Z^{2}}{K_{2}^{2}+Z^{2}}$,

$V_{3}=\beta V_{\mathrm{M} 3} \frac{Y^{2}}{K_{\mathrm{y}}^{2}+Y^{2}} \frac{Z^{4}}{K_{\mathrm{z}}^{4}+Z^{4}}$,

where $v_{0}$ denotes the passive influx of $\mathrm{Ca}^{2+}$ from the extracellular medium, $V_{\mathrm{M} 2}$ and $V_{\mathrm{M} 3}$ are, respectively, the maximum pumping rate into and the maximum rate of release of $\mathrm{Ca}^{2+}$ from the intracellular $\mathrm{Ca}^{2+}$ store and $K_{2}, K_{\mathrm{y}}$ and $K_{z}$ are the threshold constants for pumping, release and activation, respectively. All concentrations are defined with respect to the total cell volume. Furthermore, $0 \leq \beta$ $\leq 1$ represents the stimulation level which affects both the $\mathrm{Ca}^{2+}$ entry from the extracellular medium $(v, \beta)$ and the release of $\mathrm{Ca}^{2+}$ from the intracellular store $\left(V_{3}\right)$. In this version of the model, the inhibition of the channel activity at high cytosolic $\mathrm{Ca}^{2+}$ concentrations is not taken into account. This inhibition will be incorporated into the model considered in Section 3 . The effect of this negative regulation has only been studied so far in relation to simple periodic oscillations $[13,16-18,30,31]$.

\section{Bursting in the one-pool model with $\mathrm{Ca}^{2+}$ inhibition of the $\mathrm{Ca}^{2+}$ channel}

The two types of $\mathrm{Ca}^{2+}$ channels that have been identified in the $\mathrm{ER}$, i.e. the $\mathrm{Ca}^{2+}$-sensitive $\mathrm{RyR}$ channel and the $\operatorname{IP}_{3} R$ channel, both consist of tetrameric receptors [35]. The RyR channel is gated by $\mathrm{Ca}^{2+}$ and shows a bell-shaped dependence on the intracellular $\mathrm{Ca}^{2+}$ concentration [28]. The $\mathrm{IP}_{3}$-sensitive $\mathrm{Ca}^{2+}$ channel is activated in a more complex way. Binding of $\mathrm{IP}_{3}$ is required to open the $\mathrm{Ca}^{2+}$ channel $[27,40]$. Experiments show that the release of ${ }^{45} \mathrm{Ca}^{2+}$ increases as a function of the $\mathrm{IP}_{3}$ concentration [34]. However, in order to release $\mathrm{Ca}^{2+}$ from the store a second signal is required: $\mathrm{Ca}^{21}$ needs to bind to the cytosolic side of the channel [40]. Low concentrations of cytosolic $\mathrm{Ca}^{2+}$ activate the channel, thereby stimulating CICR. High concentrations, however, result in time-dependent inactivation of the channel [16,27,30,34,41-43]. Models have shown that this dynamics of the $\mathrm{IP}_{3} \mathrm{R}$ is by itself able to account for simple periodic $\mathrm{Ca}^{21}$ oscillations $[13,16,17,30,31]$.

Here we model the dynamics of the $\mathrm{Ca}^{2+}$-release channel based on a mechanism proposed by Fabiato [44] and modelled in a different manner by Tang and Othmer [18]. The channel that we consider is both activated and inactivated by cytosolic $\mathrm{Ca}^{2+}$. Assuming that the $\mathrm{IP}_{3}$ concentration in the cell is at a constant stimulatory level, the model, which is intended to be general and phenomenological, could, in a first approximation, represent both the RyR and the $I P_{3} R$ channel. If the channel represents an $I P_{3} R$ channel, binding of $\mathrm{IP}_{3}$ is assumed to be instantaneous. We assume that there are two types of $\mathrm{Ca}^{2+}$ binding sites at the cytosolic side of the channel, one for positive and the other for negative regulation. Calcium binds rapidly to the former, but at a low affinity. Calcium binding to the other, inhibitory site is relatively slow but occurs at a higher affinity [44]. For each of the binding sites we assume that the Hill coefficient is equal to 4 , i.e. $4 \mathrm{Ca}^{2+}$ molecules need to bind in order to lead to a conformational change of the channel. Cooperativity in $\mathrm{Ca}^{2+}$ activation and inhibition is indeed suggested by the fact that the $\mathrm{IP}_{3}$ receptor is a homotetramer; the existence of such a highly cooperative process has moreover received some experimental support [45]. $R$ and $R^{+}$represent 


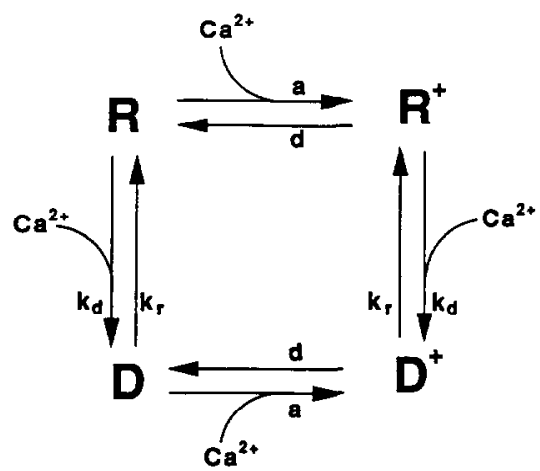

Fig. 3. Schematic representation of the dynamics of the $\mathrm{Ca}^{2+}$-rclease channel. $R$ represents the fraction of free channels, and $R^{+}$ the fraction of channels with $\mathrm{Ca}^{2+}$ bound to the activatory site. $D$ represents the fraction of channels with $\mathrm{Ca}^{2+}$ bound to the inhibitory site, and $D^{+}$the fraction of channels with $\mathrm{Ca}^{2+}$ bound to both the inhibitory and the activatory sites. The kinetic constant for activatory $\mathrm{Ca}^{2+}$ binding is called $a$, whereas $d$ represents the kinetic constant for deactivation; $k_{\mathrm{d}}$ represents the rate at which channels are inactivated and $k_{\mathrm{r}}$ denotes the rate of the reverse process.

the concentrations of free channels and of channels with $\mathrm{Ca}^{2+}$ bound to the activatory site, respectively, divided by the total channel concentration. $D$ and $D$ represent the fractions of inactivated channels, i.e. the channels with $\mathrm{Ca}^{2+}$ bound only to the inhibitory site or to both the inhibitory and the activatory sites, respectively (see Fig. 3).

Following Fabiato [44], we assume that $\mathrm{Ca}^{2+}$ binding to the activatory and inhibitory sites are two independent processes and that channels can only conduct $\mathrm{Ca}^{2+}$ from the store to the cytosol if they are in state $R^{+}$. The kinetic constant for activatory $\mathrm{Ca}^{2+}$ binding is called $a$, whereas $d$ represents the kinetic constant for deactivation. Moreover $k_{\mathrm{d}}$ characterises the rate at which channels inactivate following binding of $\mathrm{Ca}^{2+}$ to the inhibitory site, and $k_{\mathrm{r}}$ denotes the rate constant for the reverse process, i.e. the loss of $\mathrm{Ca}^{2+}$ at the inhibitory binding site. Hence, the differential equations for the different receptor states are given by:

$$
\begin{aligned}
& \frac{\mathrm{d} R}{\mathrm{~d} t}=-a R Z^{4}+\mathrm{d} R^{+}-k_{\mathrm{d}} R Z^{4}+k_{\mathrm{r}} D, \\
& \frac{\mathrm{d} R^{+}}{\mathrm{d} t}=a R Z^{4}-\mathrm{d} R^{+}-k_{\mathrm{d}} R^{+} Z^{4}+k_{\mathrm{r}} D^{+},
\end{aligned}
$$

$$
\begin{aligned}
& \frac{\mathrm{d} D}{\mathrm{~d} t}=-a D Z^{4}+\mathrm{d} D^{+}+k_{\mathrm{d}} R Z^{4}-k_{\mathrm{r}} D, \\
& \frac{\mathrm{d} D^{+}}{\mathrm{d} t}=a D Z^{4}-\mathrm{d} D^{+}+k_{\mathrm{d}} R^{+} Z^{4}-k_{\mathrm{r}} D^{+} .
\end{aligned}
$$

Because binding of $\mathrm{Ca}^{2+}$ to and release of $\mathrm{Ca}^{2+}$ from the activatory sites are fast processes, we can make a 'quasi-steady state' assumption (see [46]), writing:

$a R Z^{4}=\mathrm{d} R^{+}$,

or

$R^{+}=\gamma R$,

and

$a D Z^{4}=\mathrm{d} D^{+}$,

or

$D^{+}=\gamma D$,

where

$\gamma=\frac{a}{\mathrm{~d}} Z^{4}$.

We define $\delta$ to be the total fraction of inactivated channels and $\rho$ to be the total fraction of channels in the active $\left(R^{+}\right)$and activatable $(R)$ states, i.e.:

$\rho=R+R^{+}$,

and

$\delta=D+D^{+}$.

Now we can determine the fraction of all the states of the channel in terms of $\rho$ and $\delta$ :

$$
\begin{aligned}
& R=\frac{\rho}{1+\gamma} \\
& R^{+}=\frac{\gamma \rho}{1+\gamma},
\end{aligned}
$$

and

$D=\frac{\delta}{1+\gamma}, D^{+}=\frac{\gamma \delta}{1+\gamma}$.

Realizing that $\delta=1-\rho$, the differential equation for $\rho$ can be written:

$\frac{\mathrm{d} \rho}{\mathrm{d} t}=-k_{\mathrm{d}} Z^{4} \rho+k_{r}(1-\rho)$. 
This equation for the evolution of the fraction of active $\mathrm{Ca}^{2+}$ channels has the same form as the one used in other models for $\mathrm{Ca}^{2+}$ oscillations [18,30], in which no bursting behaviour was reported. The evolution of the fraction of active $\mathrm{Ca}^{2+}$ channels in this model is thus given by integration of Eq. (12) and substitution into $\mathrm{Eq}$. (10b). When the steady-state value of the active receptor $\left(R^{+}\right)$is plotted against the logarithm of the cytosolic $\mathrm{Ca}^{2+}$ concentration $(Z)$ the characteristic bell-shaped curve is obtained (data not shown). We now have to couple this mechanism for the bell-shaped dependence of the $\mathrm{Ca}^{2+}$ channel on cytosolic $\mathrm{Ca}^{2+}$ with the initial model defined by Eqs. (1a), (1b), (2)-(4). Because the activation of CICR by cytosolic $\mathrm{Ca}^{2+}$ has already been taken into account in Eq. (12), $V_{3}$ becomes:

$V_{3}=\beta R^{+} V_{\mathrm{M} 3} \frac{Y^{2}}{K_{y}^{2}+Y^{2}}$.

Replacing Eq. (4) by Eq. (13) in the system defined by Eqs. (1a), (1b), (2)-(4) and (12) yields a model which incorporates $\mathrm{Ca}^{2+}$ inhibition of the $\mathrm{Ca}^{2+}$ channel. The scheme of this model corresponds to Fig. 2, in which the active state of the channel becomes a variable of the model because of inhibition at high $\mathrm{Ca}^{2+}$ levels. The $\mathrm{Ca}^{2+}$-release channel in the picture represents either a RyR channel or an $\mathrm{IP}_{3} \mathrm{R}$ channel. Note that in the case it denotes a RyR channel, the increased production of $\mathrm{IP}_{3}$ which results after extracellular stimulation, will not affect CICR. Hence, the dotted part of the picture does not play any role in this case; extracellular stimulation will only affect CICR via the stimulated influx of $\mathrm{Ca}^{2+}$. In this case $\beta$ does not appear in Eq. (13). However, this will not change the results of the model qualitatively since there will still be a range of $V_{\mathrm{M} 3}$ values producing oscillations.

As expected, simple periodic oscillations in cytosolic $\mathrm{Ca}^{2+}$ are readily generated by integration of the model defined by Eqs. (1a), (1b), (2), (3), (10b), (12) and (13) (see Fig. 4). These spikes are accompanied by regular variations in the fraction $\rho$ of channels in the active $\left(R^{+}\right)$and activatable $(R)$ states. Parameter values have been chosen in order to obtain oscillations with a period of the order of $30 \mathrm{~s}$. Again, as the model is used to investigate the possibility of the existence of complex oscillations and supposed to describe both the RyR and the $\mathrm{IP}_{3} \mathrm{R}$ channel, we did not attempt to fit these values to data reported for kinetic constants, given the fact that many of these constants have not yet been precisely determined.

As shown in the timeplot of Fig. 5 bursting also arises in this model. Two phases can be distinguished. In the active phase $\mathrm{A}$ the cytosolic $\mathrm{Ca}^{2+}$ concentration oscillates around a plateau level. In this phase the spikes are generated due to the dynamics of the $\mathrm{Ca}^{2+}$-release channel. The rising phase of a spike is due to CICR, and the decreasing phase results from the pumping back of $\mathrm{Ca}^{2+}$ into the stores and into the external medium, which are not balanced by $\mathrm{Ca}^{2+}$ release any more because of $\mathrm{Ca}^{2+}$-channel inactivation. In the silent phase $\mathrm{B}$ the cytosolic $\mathrm{Ca}^{2+}$ concentration is at a basal level. Both the $\mathrm{Ca}^{2+}$ concentration in the store $(Y)$ and the fraction $\rho$ of active $\left(R^{+}\right)$and activatable $(R)$ channels increase during this phase.

The mechanism behind the transitions between the active and silent phases can be studied by exploiting the different time scales of the variables of the model [38]. In the present model, the relatively slow dynamics of $Y$ appears to modulate the fast spike-generating subsystem $(\rho, Z)$. This fast subsystem in turn influences the evolution of $Y$. Following Rinzel [38], $Y$ can be considered as a parameter

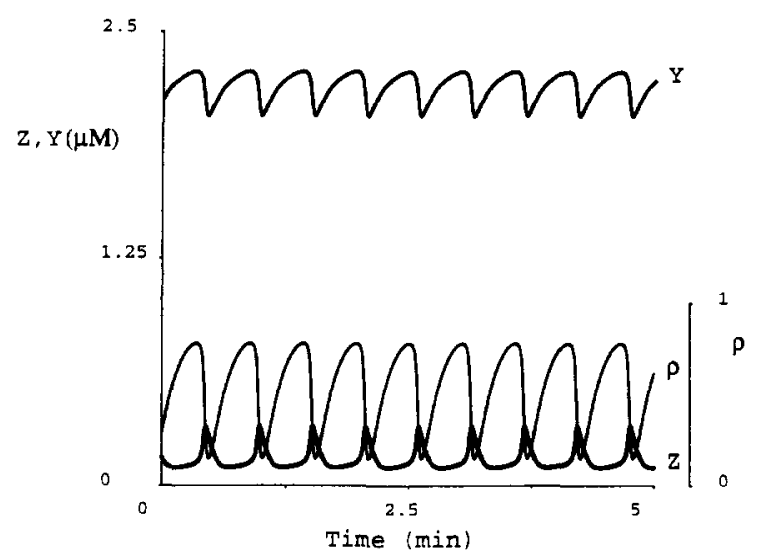

Fig. 4. Simple periodic oscillations in the CICR model with $\mathrm{Ca}^{2+}$ inhibition of the $\mathrm{Ca}^{2+}$-release channel, defined by Eqs. (1a), ( $1 \mathrm{~b}$ ), (2), (3), (10b), (12) and (13). Parameter values are: $v_{0}=v_{1}=1$ $\mu \mathrm{M} \cdot \min ^{-1}, V_{\mathrm{M} 2}=6.5 \mu \mathrm{M} \cdot \min ^{-1}, V_{\mathrm{M} 3}=50 \mu \mathrm{M} \cdot \min ^{-1}, K_{2}=$ $0.1 \mu \mathrm{M}, \quad K_{\mathrm{y}}=0.2 \mu \mathrm{M}, \quad a=4 \cdot 10^{4} \mathrm{~min}^{-1}(\mu \mathrm{M})^{-4}, d=100$ $\min ^{-1}, k_{\mathrm{d}}=5000 \min ^{-1}(\mu \mathrm{M})^{-4}, k_{\mathrm{r}}=5 \min ^{-1}, k_{\mathrm{f}}=1 \min ^{-1}$, $k=10 \mathrm{~min}^{-1}$ and $\beta=0.5$. 

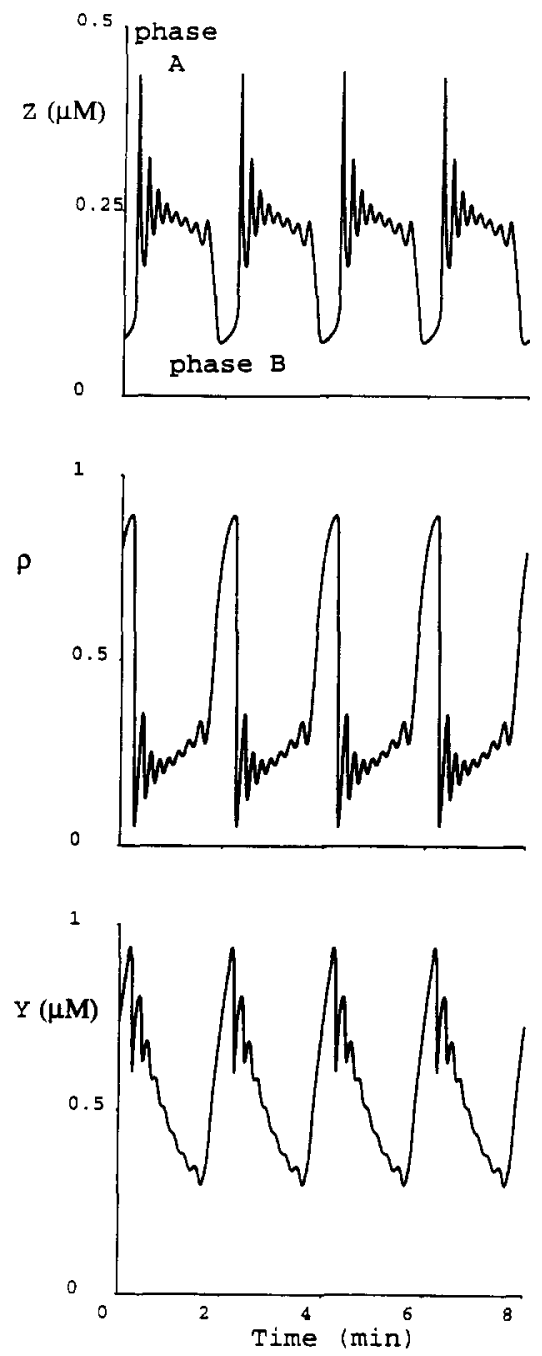

Fig. 5. $\mathrm{Ca}^{2+}$ bursting oscillations in the model based on CICR with $\mathrm{Ca}^{2+}$ inhibition of the $\mathrm{Ca}^{2+}$-release channel, defined by Eqs. (1a), (1b), (2), (3), (10b), (12) and (13). Phase A indicates the active phase during which cytosolic $\mathrm{Ca}^{2+}$ oscillates around a plateau level, while phase $B$ indicates the silent phase. Parameter values are as in Fig. 4, with $\beta=1$.

controlling the 2-variable subsystem $(\rho, Z)$. The resulting bifurcation diagram, denoted by the thick line in Fig. 6, shows the steady-state value of $Z$ and its stability properties as a function of $Y$. Stable and unstable steady states are represented by solid and dashed lines, respectively. The stable focus on the upper branch of the diagram becomes unstable through a subcritical Hopf bifurcation (denoted by $\mathrm{HB}$ in Fig. 6). The figure also shows one period of the bursting behaviour of Fig. 5 in the $(Y, Z)$ phase plane. This trajectory, denoted by the thin line, was obtained by numerical integration of the 3-variable system. As indicated by the arrows in the diagram, for high values of $Z$, i.e. on the upper branch of the bifurcation diagram, the $\mathrm{Ca}^{2+}$ concentration of the intracellular pool decreases due to CICR-induced spikes. In contrast $Y$ increases when $Z$ is at its basal level, i.e. on the lower branch of the bifurcation diagram, because replenishment of the pool supersedes CICR. The fact that the trajectory does not exactly follow the bifurcation diagram results from that the dynamics of $Y$ is not sufficiently slow to allow it to be treated as a parameter.

Fig. 6 clearly shows that the concentration of $\mathrm{Ca}^{2+}$ in the ER gradually decreases due to the $\mathrm{Ca}^{2+}$

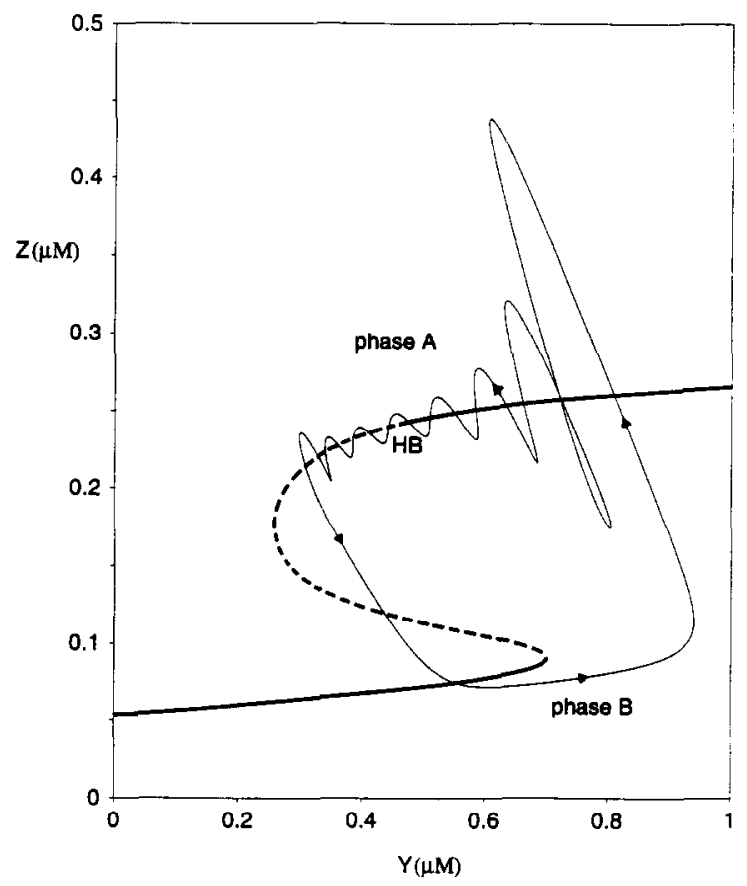

Fig. 6. $\mathrm{Ca}^{2+}$ bursting oscillations of Fig. 5 plotted in the $(Y, Z)$ phase plane. The thin line represents the trajectory, whereas the thick line denotes the bifurcation diagram (obtained by using the bifurcation programme AUTO86). Unstable steady states are represented by dashed lines and stable steady states are represented by solid lines. The subcritical Hopf bifurcation is denoted by HB. The arrows indicate the direction of the trajectory. 
oscillations between the ER and the cytosol (phase A). As a consequence, the release of $\mathrm{Ca}^{2+}$ from the pool decreases, and the level of $Z$ finally falls below the threshold for CICR. The oscillations stop and the cytosolic $\mathrm{Ca}^{2+}$ concentration returns to its basal level; the ER is then refilled thanks to the entry of extracellular $\mathrm{Ca}^{2+}$ which is immediately pumped from the cytosol into the store (phase B). As soon as the threshold for CICR is reached, spiking resumes. The reason why bursting occurs instead of simple periodic oscillations is that the channel inactivation at high $Z$ levels postpones the depletion of the $\mathrm{Ca}^{2+}$ pool (in the absence of channel inhibition by $Z$, this depletion of the $\mathrm{Ca}^{2+}$ pool together with the extrusion of $\mathrm{Ca}^{2+}$ from the cytosol due to $\mathrm{Ca}^{2+}$ pumps provoke the termination of a spike and produce simple periodic oscillations). A series of small-amplitude spikes in $Z$ consequently occurs, until the level of $Y$ has sufficiently dropped so that $Z$ falls below the threshold for CICR.

\section{Bursting and chaos in the one-pool model with $\mathrm{Ca}^{2+}$-activated $\mathrm{IP}_{3}$ degradation}

In Section 3 we have implicitly assumed a constant stimulatory intracellular $\mathrm{IP}_{3}$ concentration when considering the case of the $\mathrm{IP}_{3} \mathrm{R}$ channel. This assumption is corroborated by experiments which showed that $\mathrm{IP}_{3}$-dependent $\mathrm{Ca}^{2+}$ oscillations can arise in the absence of any variations in the $\mathrm{IP}_{3}$ level [47]. However, the $\mathrm{IP}_{3}$ concentration most probably varies in the course of time. Meyer and Stryer [7] proposed a theoretical model for simple periodic $\mathrm{Ca}^{2+}$ oscillations based on the activation of $\mathrm{IP}_{3}$ synthesis by cytosolic $\mathrm{Ca}^{2+}$. Complex oscillations of $\mathrm{Ca}^{2+}$ have recently been ohserved in an extension of this model by Shen and Larter [32]. Here we focus on the well-documented effect of cytosolic $\mathrm{Ca}^{2+}$ on the degradation of $\mathrm{IP}_{3}$ (see for example [36]). This effect was taken into account in the model for simple periodic $\mathrm{Ca}^{2+}$ oscillations proposed by Swillens and Mercan [9]. Because $\mathrm{Ca}^{2+}$ and $\mathrm{IP}_{3}$ act as co-agonists to stimulate $\mathrm{Ca}^{2+}$ release through the $\mathrm{IP}_{3} \mathrm{R}$ channel, we investigated the possibility that bursting can arise as a result of the $\mathrm{Ca}^{2+}$ activation of $\mathrm{IP}_{3}$ degradation.

To take into account the role of $\mathrm{IP}_{3}$ as co-agonist, we let the term for CICR be dependent on the $\mathrm{IP}_{3}$ concentration $(A)$, writing:

$V_{3}=V_{\mathrm{M} 3} \frac{A^{4}}{K_{\mathrm{a}}^{4}+A^{4}} \frac{Y^{2}}{K_{\mathrm{y}}^{2}+Y^{2}} \frac{Z^{4}}{K_{\mathrm{z}}^{4}+Z^{4}}$,

where $K_{\mathrm{a}}$ represents the threshold constant for activation by $\mathrm{IP}_{3}$, which is considered as a cooperative process, in agreement with experimental observations [48]. The dynamics of $\mathrm{IP}_{3}$ is modelled in the following way:

$\frac{\mathrm{d} A}{\mathrm{~d} t}=\beta v_{\mathrm{p}}-v_{\mathrm{d}} \frac{A^{2}}{K_{\mathrm{p}}^{2}+A^{2}} \frac{Z^{n}}{K_{\mathrm{d}}^{n}+Z^{n}}-\epsilon A$,

where $\beta v_{\mathrm{p}}$ denotes the agonist-stimulated production of $\mathrm{IP}_{3}, v_{\mathrm{d}}$ represents the maximum rate of $\mathrm{IP}_{3}$ degradation stimulated by cytosolic $\mathrm{Ca}^{2+}$, and $K_{\mathrm{p}}$ and $K_{\mathrm{d}}$ are, respectively, the threshold constants for $\mathrm{IP}_{3}$ degradation and for $\mathrm{Ca}^{2+}$ activation of $\mathrm{IP}_{3}$ degradation. The cooperativity in the effect of cytosolic $\mathrm{Ca}^{2+}$ holds with the observation that $\mathrm{Ca}^{2+}$ action is mediated by calmodulin [36]. Moreover $\epsilon$ represents the $\mathrm{Ca}^{2+}$-independent decay of $\mathrm{IP}_{3}$, which is particularly important if the cytosolic $\mathrm{Ca}^{2+}$ concentration is very low. Replacing Eq. (4) by Eq. (14) in the basic CICR model and adding Eq. (15) yields the model with $\mathrm{Ca}^{2+}$-activated $\mathrm{IP}_{3}$ degradation (see Fig. 7).

For $\beta=0.4$ the model gives rise to simple periodic oscillations. For larger valucs of stimulation, bursting arises (Fig. 8). As shown in Fig. 9, the

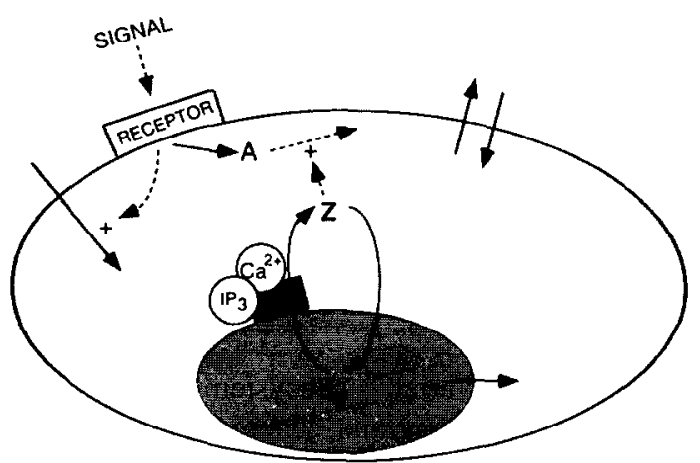

Fig. 7. Schematic representation of the one-pool model based on CICR with $\mathrm{Ca}^{2+}$-stimulated degradation of $\mathrm{IP}_{3}(A), Z$ and $Y$ represent cytosolic and intravesicular $\mathrm{Ca}^{2+}$, respectively. Solid arrows denote $\mathrm{Ca}^{2+}$ fluxes. A dotted arrow represents the degradation of $\mathrm{IP}_{3}$, which is stimulated by $Z$ (see text for details). 
mechanism of bursting is quite similar to the one described for the model with $\mathrm{Ca}^{2+}$-channel inactivation (compare Fig. 9 with the corresponding Fig. 6 obtained for the latter model). At a high level of intravesicular $\mathrm{Ca}^{2+}$, fast spikes in $Z$ occur (phase A). The rising phase of each spike is due to CICR;
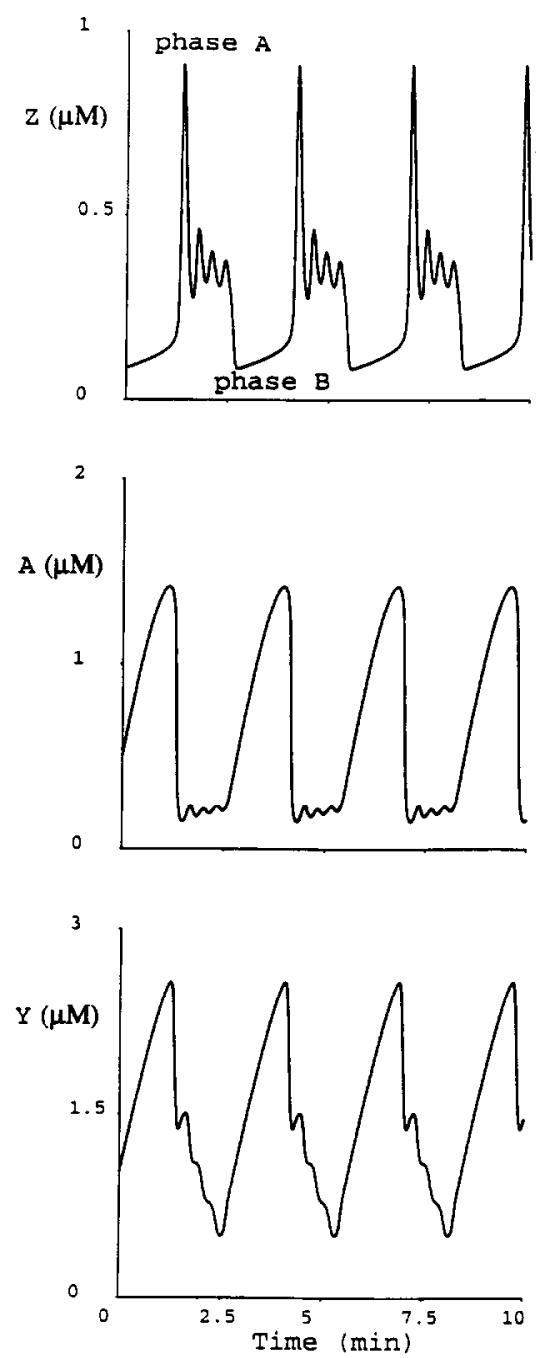

Fig. 8 . Bursting in the model with $\mathrm{Ca}^{2+}$-activated degradation of $\mathrm{IP}_{3}$, defined by Eqs. (1a), (1b), (2), (3), (14) and (15). Parameter values are: $v_{0}=2 \mu \mathrm{M} \cdot \min ^{-1}, v_{1}=1 \mu \mathrm{M} \cdot \min ^{-1}, V_{\mathrm{M} 2}=6.5$ $\mu \mathrm{M} \cdot \min ^{-1}, V_{\mathrm{M} 3}=19.5 \mu \mathrm{M} \cdot \mathrm{min}^{-1}, K_{2}=0.1 \mu \mathrm{M}, K_{\mathrm{a}}=0.2 \mu \mathrm{M}$, $K_{y}=0.2 \mu \mathrm{M}, K_{z}=0.3 \mu \mathrm{M}, v_{\mathrm{p}}=2.5 \mu \mathrm{M} \cdot \min ^{-1}, v_{\mathrm{d}}=80$ $\mu \mathrm{M} \cdot \min ^{-1}, K_{\mathrm{p}}=1 \mu \mathrm{M}, K_{\mathrm{d}}=0.4 \mu \mathrm{M}, \epsilon=0.1, k_{\mathrm{f}}=1 \mathrm{~min}^{-1}$, $k=10 \mathrm{~min}^{-1}, n=4$ and $\beta=0.5$.

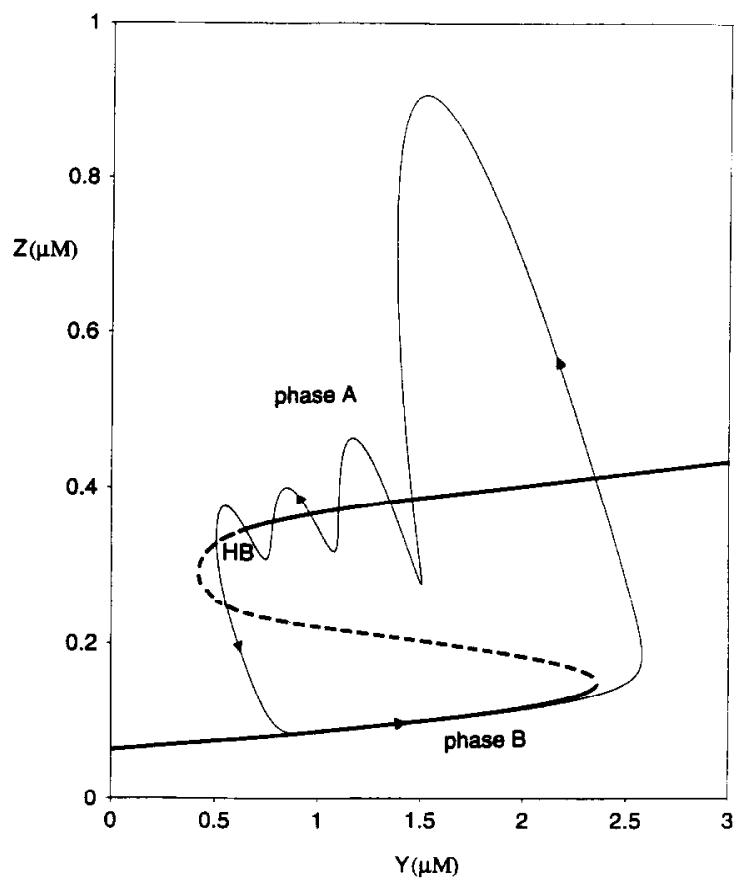

Fig. 9. $\mathrm{Ca}^{2+}$ bursting oscillations of Fig. 8 plotted in the $(Y, Z)$ phase plane. The signification of the lines is defined as in Fig. 6.

the decreasing phase is brought about by $\mathrm{Ca}^{2+}$ activation of $\mathrm{IP}_{3}$ degradation. As in the model based on $\mathrm{Ca}^{2+}$-channel inactivation, the $\mathrm{Ca}^{2+}$ concentration in the ER progressively decreases in the course of spiking, leading to the switch from the active to the silent phase. Then the concentration of $\mathrm{Ca}^{2+}$ in the store progressively increases (phase $\mathrm{B}$ ); when a threshold level of cytosolic $\mathrm{Ca}^{2+}$ is reached, a new active phase of bursting occurs.

In a rather small range of parameter values the model with $\mathrm{IP}_{3}$ degradation can also give rise to chaotic behaviour. Fig. 10a shows the chaotic variation of $Z$ as a function of time (the corresponding aperiodic variation of $Y$ and $A$ is not shown). The fact that the behaviour is indeed chaotic is demonstrated by the return map of Fig. 10b. In this figure the successive maxima of cylosolic $\mathrm{Ca}^{2+}(Z)$ are plotted as a function of their preceding maxima. If these maxima are plotted for an infinitely long time series, a continuous curve is obtained, which is characteristic of chaos. A classical sequence of period-doubling bifurcations leading from simple peri- 
(a)

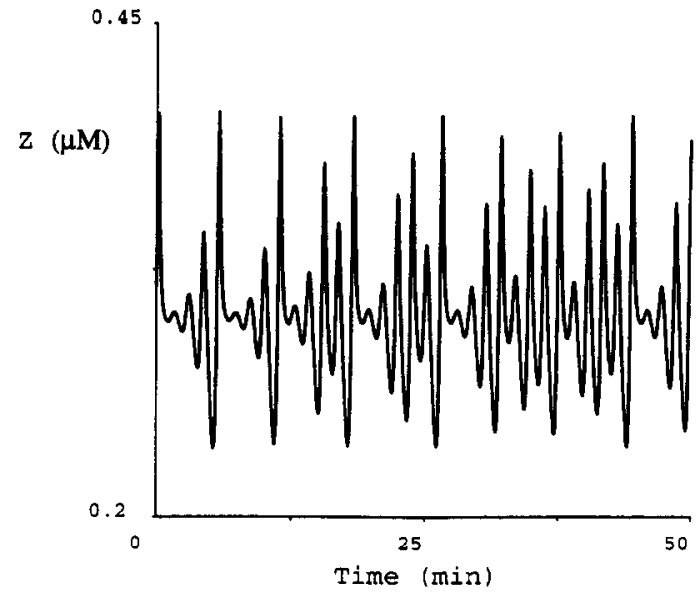

(b)

(b) 0.42

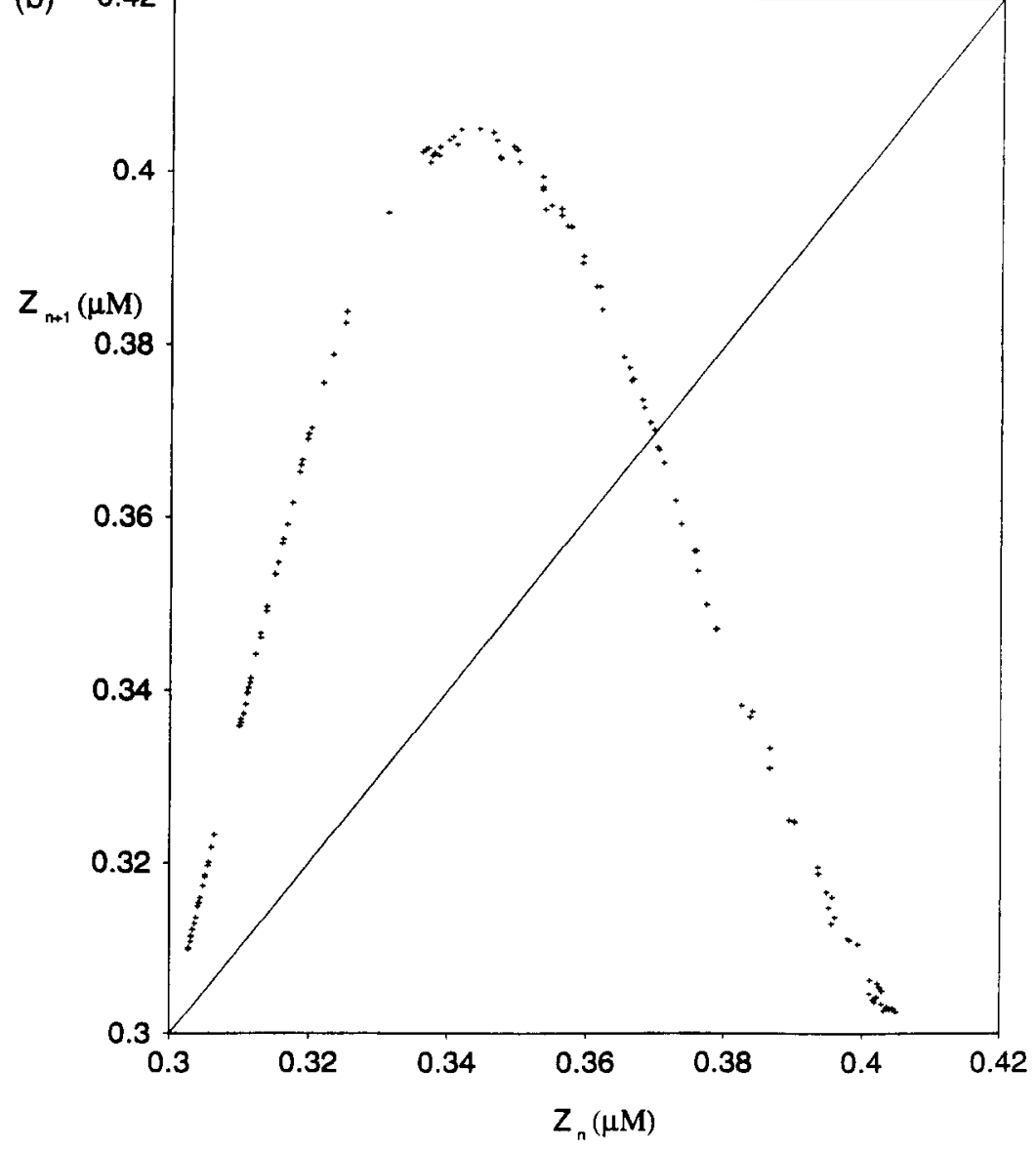


odic behaviour to chaos is observed as a function of parameter $\beta$, which gives another indication that the oscillatory behaviour shown in Fig. 10a is indeed chaotic (data not shown).

\section{Bursting in a model based on CICR with two $\mathrm{Ca}^{2+}$ pools}

The first version of the CICR model [8] takes two different intracellular $\mathrm{Ca}^{2+}$ stores into account. One pool is sensitive to $\mathrm{Ca}^{2+}$ but insensitive to $\mathrm{IP}_{3}$, the other one is sensitive to $\mathrm{IP}_{3}$. These different $\mathrm{Ca}^{2+}$ stores have been identified in adrenal chromaffin cells [49-51], pituitary cells [52], Purkinje neurons [53], parotid acinar cells [54] and in vascular smooth muscle cells [55]. The $\mathrm{Ca}^{2+}$ dependence of the activity of the $\mathrm{IP}_{3}$-sensitive channel, unknown at that time, was not incorporated in the model. This situation, somewhat unrealistic from a physiological point of view, has been retained here, mainly because it allowed us to uncover an interesting way of generating complex oscillations. In the original version of the two-pool model the $\mathrm{Ca}^{2+}$ concentration in the $\mathrm{IP}_{3}$-sensitive pool was assumed to remain constant. We are currently investigating the possible occurrence of bursting in an extended model in which the $\mathrm{Ca}^{2+}$ dependence of the $\mathrm{IP}_{3}$ receptor in the $\mathrm{IP}_{3}$ sensitive pool is taken into account. Here we show that bursting can occur in this model if the $\mathrm{Ca}^{2+}$ concentration in that pool is allowed to vary.

The $\mathrm{Ca}^{2+}$-exchange processes between the extracellular medium, the cytosol and the $\mathrm{Ca}^{2+}$-sensitive pool are described as in a previous publication [8]. Moreover, we assume here that the $\mathrm{Ca}^{2+}$ concentration in the $\mathrm{IP}_{3}$-sensitive pool $(X)$ decreases due to a passive $\mathrm{Ca}^{2+}$ leak and due to $\mathrm{IP}_{3}$-activated release into the cytosol. The $\mathrm{Ca}^{2+}$ concentration in this pool increases due to pumping of $\mathrm{Ca}^{2+}$ from the cytosol

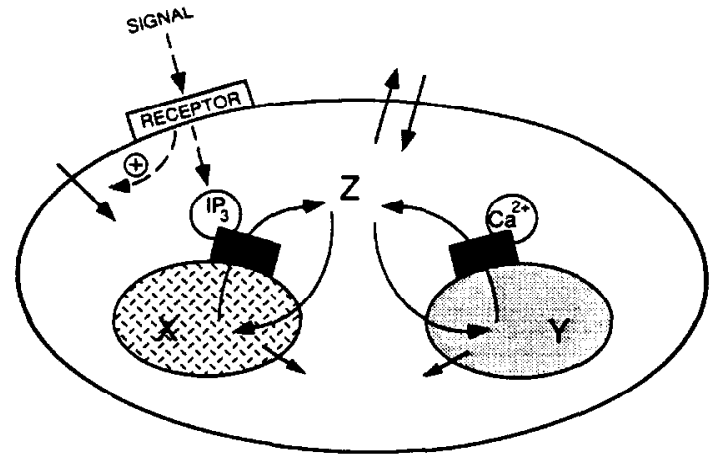

Fig. 11. Schematic representation of the two-pool model for complex $\mathrm{Ca}^{2+}$ oscillations. The $X$ pool is sensitive to $\mathrm{IP}_{3}$, whereas the $Y$ pool is sensitive to $\mathrm{Ca}^{2+}$. Solid arrows denote $\mathrm{Ca}^{2+}$ fluxes (see text for details).

into the store (see Fig. 11). The equations of the model are given by:

$\frac{\mathrm{d} Z}{\mathrm{~d} t}=V_{\mathrm{in}}-V_{2 \mathrm{i}}+V_{3 \mathrm{i}}+k_{\mathrm{f}} Y-V_{2 \mathrm{~s}}+V_{3 \mathrm{~s}}+k_{\mathrm{f}} X-k Z$,

$\frac{\mathrm{d} Y}{\mathrm{~d} t}=V_{2 \mathrm{i}}-V_{3 \mathrm{i}}-k_{\mathrm{f}} Y$,

$\frac{\mathrm{d} X}{\mathrm{~d} t}=V_{2 \mathrm{~s}}-V_{3 \mathrm{~s}}-k_{\mathrm{f}} X$,

with:

$V_{\text {in }}=v_{0}+v_{1} \beta$,

$V_{2 \mathrm{i}}=V_{\mathrm{M} 2 \mathrm{i}} \frac{Z^{2}}{K_{2 \mathrm{i}}^{2}+Z^{2}}$,

$V_{3 \mathrm{i}}=V_{\mathrm{M} 3 \mathrm{i}} \frac{Y^{2}}{K_{3 y}^{2}+Y^{2}} \frac{Z^{2}}{K_{3 z}^{2}+Z^{2}}$,

Fig. 10. Chaos in the CICR model with $\mathrm{Ca}^{2+}$-activated degradation of $\mathrm{IP}_{3}$. Parameter values are: $v_{0}=2 \mu \mathrm{M}_{\mathrm{min}}{ }^{-1}, v_{1}=1 \mu \mathrm{M} \cdot \mathrm{min}^{-1}$, $V_{\mathrm{M} 2}=6.5 \mu \mathrm{M} \cdot \min ^{-1}, V_{\mathrm{M} 3}=19 \mu \mathrm{M} \cdot \min ^{-1}, K_{2}=0.1 \mu \mathrm{M}, K_{\mathrm{a}}=0.4 \mu \mathrm{M}, K_{\mathrm{y}}=0.2 \mu \mathrm{M}, K_{\mathrm{z}}=0.5 \mu \mathrm{M}, v_{\mathrm{p}}=5 \mu \mathrm{M} \cdot \mathrm{min}^{-1}, v_{\mathrm{d}}=160$ $\mu \mathrm{M} \cdot \min ^{-1}, K_{\mathrm{p}}=2 \mu \mathrm{M}, K_{\mathrm{d}}=0.4 \mu \mathrm{M}, \epsilon=0.1, k_{\mathrm{f}}=1 \mathrm{~min}^{-1}, k=10 \mathrm{~min}^{-1}, n=2$ and $\beta=1$. (a) Evolution of cytosolic Ca ${ }^{2+}(Z)$ as a function of time. (b) Analysis of the chaotic behaviour by means of a return map. The successive maxima of cytosolic Ca ${ }^{2+}(Z)$ are plotted as a function of their preceding maxima. 
(a)
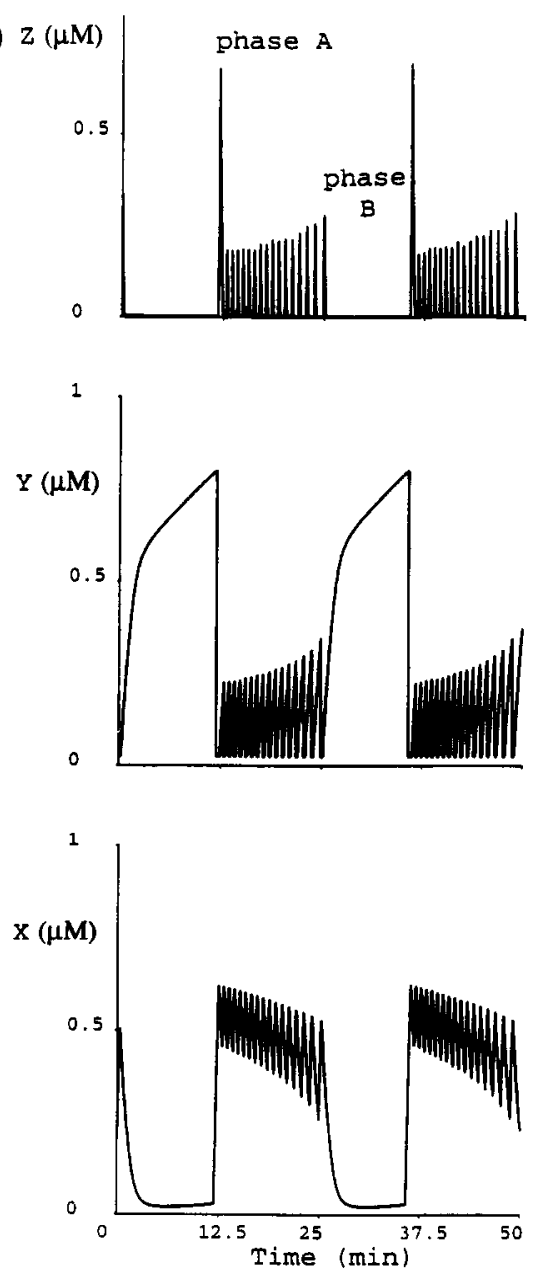

(b)

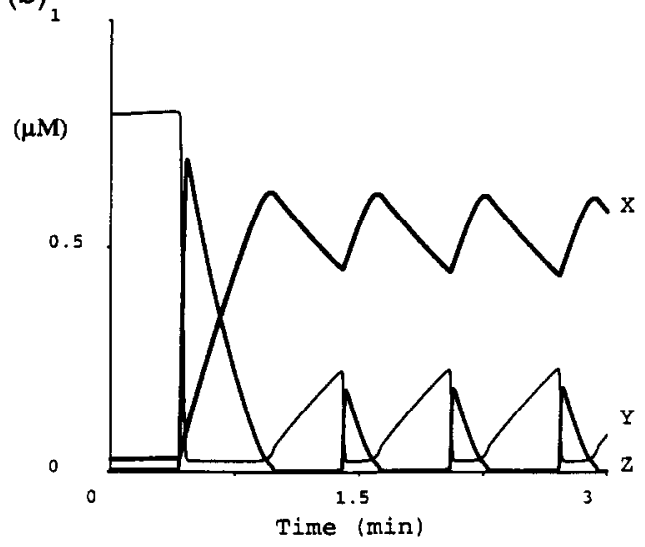

$$
V_{2 \mathrm{~s}}=V_{\mathrm{M} 2 \mathrm{~s}} \frac{Z^{2}}{K_{2 \mathrm{~s}}^{2}+Z^{2}},
$$

$$
V_{3 \mathrm{~s}}=\beta V_{\mathrm{M} 3 \mathrm{~s}} \frac{X^{2}}{K_{3 \mathrm{~s}}^{2}+X^{2}} \text {, }
$$

where $V_{2 \mathrm{i}}, V_{3 \mathrm{i}}, V_{2 \mathrm{~s}}$ and $V_{3 \mathrm{~s}}$ represent the rates of $\mathrm{Ca}^{2+}$ pumping into and release of $\mathrm{Ca}^{2+}$ from the $\mathrm{IP}_{3}$-insensitive and -sensitive pools, respectively, with $V_{\mathrm{M} 2 \mathrm{i}}, V_{\mathrm{M} 3 \mathrm{i}}, V_{\mathrm{M} 2 \mathrm{~s}}$ and $V_{\mathrm{M} 3 \mathrm{~s}}$ denoting the maximum velocities of these processes; $K_{2 \mathrm{i}}, K_{3 \mathrm{y}}, K_{2 \mathrm{~s}}$ and $K_{3 \mathrm{~s}}$ represent the threshold constants associated with these processes. Moreover $K_{3 z}$ is the activation constant for CICR in the $\mathrm{IP}_{3}$-insensitive pool. The other constants are defined as in previous sections. All concentrations are again defined with respect to the total cell volume.

Fig. 12 shows a typical complex oscillatory pattern to which the model gives rise. The main difference between this time pattern and the ones generated by the models of Sections 3 and 4 pertains to the cytosolic $\mathrm{Ca}^{2+}$ concentration during the active phase. In Sections 3 and 4 the fast cytosolic $\mathrm{Ca}^{2+}$ oscillations occurred around a plateau level. This plateau level is not present here. Instead, during the spiking phase $Z$ oscillates around its basal level.

The parameters are chosen such that the $Y$ pool takes up $\mathrm{Ca}^{2+}$ from the cytosol at a higher velocity than the $X$ pool. Therefore, during the silent phase $X$ decreases while $Y$ increases (see phase B in Fig. 12a). When the threshold for CICR is exceeded in the cytosol, a large release of $\mathrm{Ca}^{2+}$ from the $Y$ pool occurs. Calcium which is pumped back into the $Y$ pool is then immediately released again through CICR, allowing the $\mathrm{Ca}^{2+}$ pump in the $X$ pool to become active. Therefore at each spike in $Z, Y$ decreases down to a basal level while $X$ increases.

Fig. 12. (a) Bursting in the two-pool model based on CICR, defined by Eqs. (16a), (16b), (16c), (17)-(21). Parameter values are: $v_{0}=0.015 \mu \mathrm{M} \cdot \mathrm{min}^{-1}, v_{1}=0.012 \mu \mathrm{M} \cdot \min ^{-1}, V_{\mathrm{M} 2 i}=3.1$ $\mu \mathrm{M} \cdot \mathrm{min}^{-1}, V_{\mathrm{M} 3 i}=25 \mu \mathrm{M} \cdot \mathrm{min}^{-1}, V_{\mathrm{M} 2 s}=1.5 \mu \mathrm{M} \cdot \mathrm{min}^{-1}, V_{\mathrm{M} 3 s}$ $=0.169 \mu \mathrm{M} \cdot \mathrm{min}^{-1}, K_{2 i}=0.005 \mu \mathrm{M}, K_{3 y}=0.065 \mu \mathrm{M}, K_{3 z}=$ $0.022 \mu \mathrm{M}, K_{2 s}=0.0265 \mu \mathrm{M}, K_{3 s}=0.1 \mu \mathrm{M}, k_{\mathrm{f}}=0.5 \mathrm{~min}^{-1}$, $k=1 \mathrm{~min}^{-1}$ and $\beta=1$. (b) Enlargement of the first four spikes of the active phase. At each spike there is a $\mathrm{Ca}^{2+}$ flux from the $Y$ pool via the cytosol to the $X$ pool, while between two spikes this process is reversed. 
The process repeats itself and at each spike there is some $\mathrm{Ca}^{2+}$ transfer from the $Y$ pool via the cytosol to the $X$ pool, while between two spikes $X$ is released and taken up via $Z$ into the $Y$ pool (Fig. 12b). However, at each cycle a net transfer of $\mathrm{Ca}^{2+}$ from the $X$ to the $Y$ pool occurs. The $\mathrm{Ca}^{2+}$ concentration in the $X$ pool gradually decreases to a point at which the $\mathrm{Ca}^{2+}$ input from this pool into the cytosol becomes too low to sustain the CICR process; the system then enters a silent phase during which the $\mathrm{Ca}^{2+}$ concentrations in the cytosol and in the $Y$ pool slowly increase due to the influx of $\mathrm{Ca}^{2+}$ from the extracellular medium.

Bursting in this model relies on a more complex mechanism than in the models of Sections 3 and 4 . In the present model it is difficult to identify a slower variable that switches the fast spikes on and off. Therefore it is difficult to explain bursting by means of a single bifurcation diagram as was done for previous models in Figs. 6 and 9. However, some insight into the mechanism of bursting in this model can be gained by considering two bifurcation diagrams.

Fig. 13 shows a bifurcation diagram which has been obtained by considering $X$ as a parameter controlling the 2-variable subsystem $(Y, Z)$. The thick line in this diagram shows the steady-state level of $Z$ and its stability as a function of $X$. The thin line represents the burst trajectory obtained by numerical integration of the 3-variable system. There is only one steady state, which is stable for large values of $X$ (solid line) and unstable for small values of $X$ (dashed line). The stability is changed at a subcritical Hopf bifurcation (denoted by HB in Fig. 13). At this Hopf bifurcation an unstable limit cycle arises, of which the maxima and minima are denoted by the open circles; the maxima and minima of the stable limit cycle are represented by the filled circles. As is apparent from the diagram in Fig. 13, the maxima and minima of the spikes in $Z$ closely follow the envelope of the limit cycle obtained by treating $X$ as a slowly-varying parameter.

In a burst, a first, large-amplitude spike in $Z$ (denoted by 1 in Fig. 13) is followed by a series of smaller spikes, the amplitude of which progressively increases (see Fig. 12) from the first such spike to the last, denoted respectively by 2 and $n$ in Fig. 13 . During the active phase, the $\mathrm{IP}_{3}$-sensitive pool $(X)$ is responsible for keeping $Z$ above the threshold for CICR. From Figs. 12 and 13 we see that during this phase the mean level of $X$ diminishes. This progressively hinders the accumulation of $Z$ up to the threshold for CICR. Fig. 13 clearly shows that the slope of the rise in $Z$ after a spike progressively decreases in the course of spiking. The active phase stops when $Z$ fails to reach the threshold for CICR (see phase $B$ in Figs. 12 and 13). Then $X$ and $Z$ further drop while $Y$ is replenished at their expense.

The reason for the first new spike generated after a silent phase can not be understood from Fig. 13. Therefore we consider a second bifurcation diagram (Fig. 14), in which $Y$ is a parameter controlling the 2-variable subsystem $(X, Z)$. The diagram clearly shows that during the silent phase $\mathrm{B} \mathrm{Ca}^{2+}$ is pumped into the $Y$ pool. When $Y$ attains its maximum level (denoted by ${ }^{*}$ in Fig. 14), which corresponds to the limit point of the steady-state curve of $Z$, a large

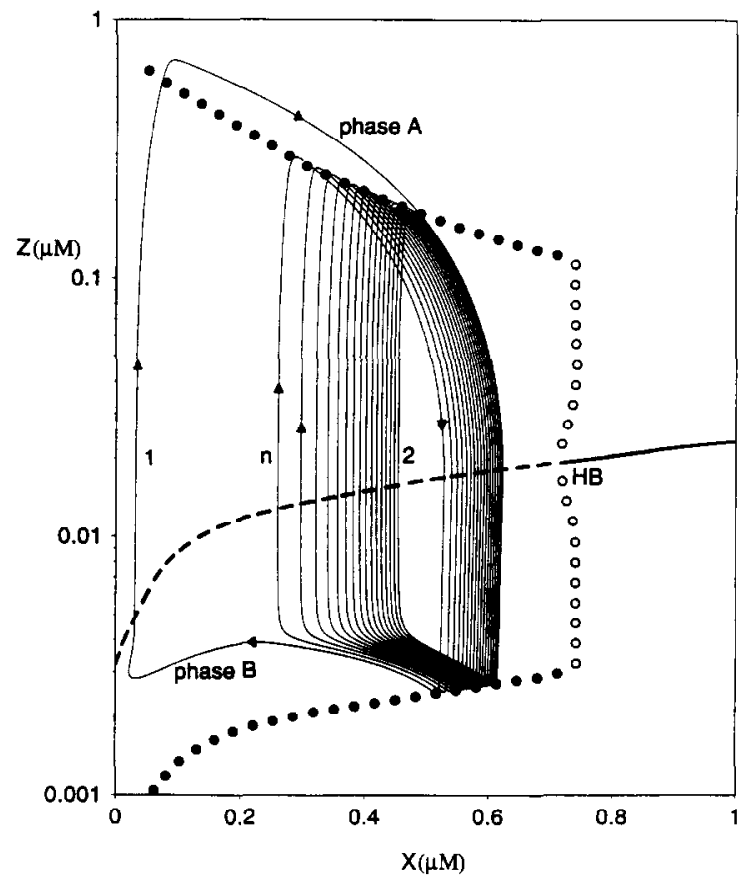

Fig. 13. $\mathrm{Ca}^{2+}$ bursting oscillations of Fig. 12 plotted in the $(X, Z)$ phase plane. The first and second spikes in $Z$ of the active phase are denoted by 1 and 2 , respectively, while the last one is denoted by $n$. The bifurcation diagram has been obtained by considering $X$ as a parameter. The unstable limit cycle which originates from the sub-critical Hopf bifurcation (HB) is denoted by the open circles; stable limit cycles are denoted by filled circles. 


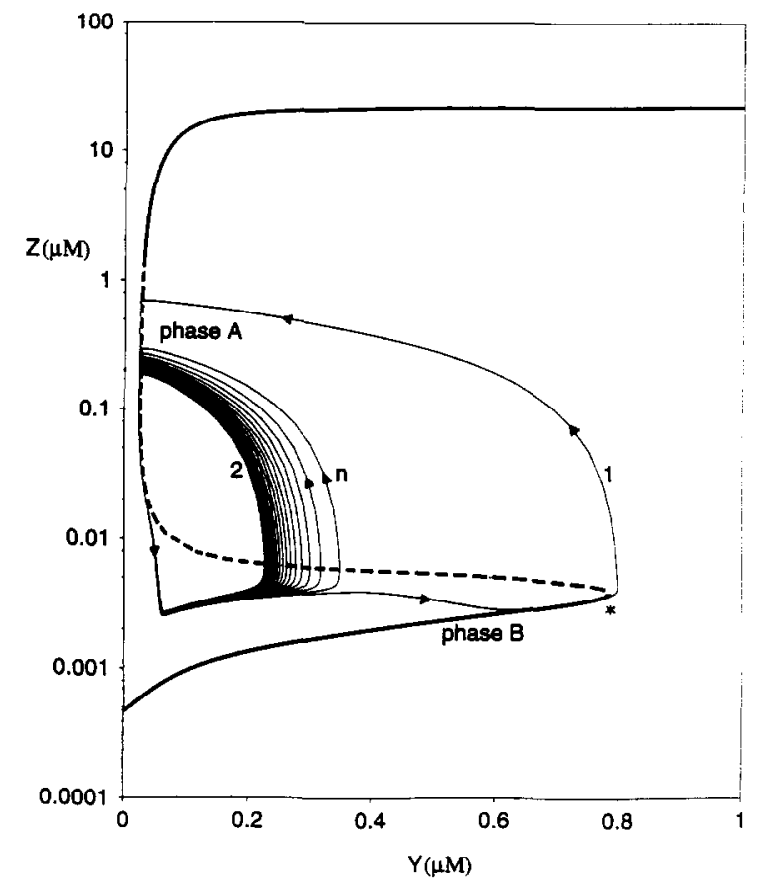

Fig. 14. $\mathrm{Ca}^{2+}$ bursting oscillations of Fig. 12 plotted in the $(Y, Z)$ phase plane. The bifurcation diagram has been obtained by considering $Y$ as a parameter. A first large spike in $Z$ is generated when $Y$ has reached its maximal level, denoted by * (see text for details).

spike in $Z$ is generated, signalling the beginning of the active phase.

The bifurcation diagrams shown in Figs. 13 and 14 provide different, complementary information. When $X$ is treated as a slowly-varying parameter, oscillations develop in the $(Z, Y)$ subsystem because of CICR, so that a Hopf bifurcation associated with a branch of limit cycles is found as a function of $X$. In contrast, when $Y$ is treated as a slowly-varying parameter, bistability occurs in the $(Z, X)$ subsystem because when $Y$ is held constant CICR can only produce bistability.

\section{Discussion}

This study is a theoretical attempt to identify possible mechanisms that could account for complex $\mathrm{Ca}^{2+}$ oscillations. Such oscillations are sometimes observed in experiments (see Fig. 1). Simple periodic oscillations of $\mathrm{Ca}^{2+}$ appear to be more com- mon, but it is conceivable that complex oscillations may sometimes be discarded because experimental reports tend to focus on simple oscillatory behaviour. Although the biological significance of $\mathrm{Ca}^{2+}$ bursting remains to be established, it is interesting from both a physiological and a theoretical point of view to understand the origin of such behaviour.

We have developed three models that can account for both simple periodic and complex $\mathrm{Ca}^{2+}$ oscillations. The first two models are based on a one-pool model based on CICR [15]. One of these models considers the $\mathrm{Ca}^{2+}$-induced inactivation of the $\mathrm{Ca}^{2+}$-release channels of the pool. The other one considers the degradation of $\mathrm{IP}_{3}$, stimulated by cytosolic $\mathrm{Ca}^{2+}$. The mechanisms for bursting in these two models are quite similar. Fast oscillations are accompanied by $\mathrm{Ca}^{2+}$-induced variations in either the fraction of active $\mathrm{Ca}^{2+}$-release channels or the concentration of $\mathrm{IP}_{3}$, which both control $\mathrm{Ca}^{2+}$ release. In both models the switch to the silent phase is due to the fact that the intravesicular $\mathrm{Ca}^{2+}$ concentration $Y$ decreases and hence does not sustain the cytosolic $\mathrm{Ca}^{2+}$ concentration above the threshold for CICR. The third model considered is an extension of a two-pool model based on CICR [8]. Here bursting arises due to the interplay between the variations of $\mathrm{Ca}^{2+}$ in the two pools.

The evolution of the cytosolic $\mathrm{Ca}^{2+}$ concentration in the first two models is qualitatively more similar to the experimental data than in the two-pool model. In the experiments on $\mathrm{Ca}^{2+}$ bursting [19-26] a switch from the silent phase to the active phase is always accompanied by a switch from a basal to an elevated level of cytosolic $\mathrm{Ca}^{2+}$. This switch is not present in the two-pool model. Instead in this model the cytosolic $\mathrm{Ca}^{2+}$ concentration oscillates around its basal level. The reason for this difference can be understood when resorting to phase-plane analysis; the latter indeed indicates that in the $(Z, Y)$ plane of the one-pool models (Figs. 6 and 9) bursting involves a movement along an $S$-shaped nullcline of which the upper branch admits damped oscillations. In contrast, such a situation does not occur in the third model, where the steady state on the upper branch in Fig. 14 is a stable node (compare with Figs. 6 and 9).

Rinzel [38], followed by Bertram et al. [56], have attempted to classify bursting oscillations into different topological and phenomenological types. Accord- 
ing to their definitions, it appears that bursting in models 1 (Fig. 5) and 2 (Fig. 8) considered here belong to a bursting mechanism of Type 1 , as they involve high frequency spikes on the top of a plateau phase. In contrast, it appears that bursting generated by model 3 (Fig. 12) does not fall clearly into one of the categories proposed by these authors. Similarly to another type of bursting reported by Rinzel [38], this system shows the existence of a sub-critical Hopf bifurcation. However, the latter bifurcation point is never crossed during bursting. Moreover, as clearly emphasized by Fig. 14, bistability in the $Z-X$ fast sub-system clearly plays an important role.

The occurrence of bursting and chaos is favoured by the interplay between two instability-generating mechanisms [37], like in a model for cAMP signalling in Dictyostelium [57] and in a model based on coupled autocatalytic enzyme reactions [39]. Two oscillatory mechanisms coupled in parallel can accordingly be identified in models 1 and 2 . In these models, a single destabilizing process, CICR, is coupled to two distinct processes limiting this selfamplification. The emptying of the $\mathrm{Ca}^{2+}$ pool is one of these processes; desensitization of the $\mathrm{IP}_{3}$ receptor or activation by $\mathrm{Ca}^{2+}$ of $\mathrm{IP}_{3}$ degradation is the second limiting process in models 1 and 2 , respectively. That an interplay between two oscillatory mechanisms is not a prerequisite for complex oscillatory phenomena, however, is exemplified by model 3 in which a single oscillatory mechanism due to CICR in the $\mathrm{Ca}^{2+}$-sensitive pool is modulated by the variable input of $\mathrm{Ca}^{2+}$ from the $\mathrm{IP}_{3}$-sensitive pool.

Experimentally, single periodic oscillations or bursting have both been observed in single hepatocytes (see Fig. 1), but what biochemical parameter controls the 'choice' between the two modes of oscillatory behaviour remains unclear. The selection of either simple or complex oscillations depends on the cell that is stimulated, at a given agonist level [P. Cobbold, personal communication]. Clearly, distinct cells are expected to vary with respect to a large number of biochemical parameters which play a role in $\mathrm{Ca}^{2+}$ signalling. To illustrate but one way to switch from simple to complex oscillations, we show in Fig. 15 how the continuous variation in the level of stimulation, measured by parameter $\beta$, can induce the transition from simple periodic oscillations to bursting in one of the models considered here. It

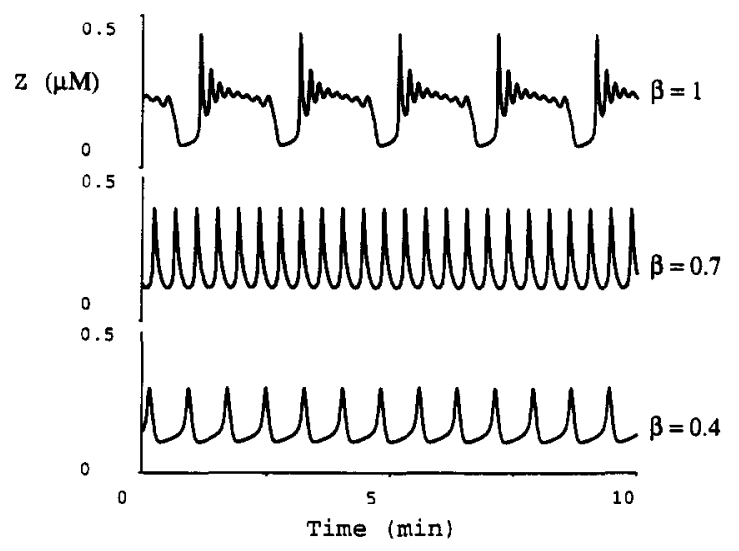

Fig. 15. A change in the stimulation level $(\beta)$ leads to a transition from simple periodic oscillations to bursting oscillations. Moreover, the frequency of the simple periodic oscillations increases with the stimulation level. Equations and parameters are similar to Fig. 4 , except for $\beta$ which is varying as shown in the figure.

should be interesting to determine whether such a transition can occur when the same hepatocyte is submitted to stimuli of varying magnitude.

Bursting is observed with certain agonists but not with others in a given cell type. Thus, in hepatocytes, only simple oscillations can be elicited by ADP whereas either simple or complex oscillations may be induced by ATP [25]. According to Dixon et al. [25], this variety in temporal patterns could arise because of differences in the negative feedback exerted by PKC or $\mathrm{G}$ proteins coupled to the receptors. For these authors, PKC would be responsible for the termination of a spike in the case of simple $\mathrm{Ca}^{2+}$ oscillations, while regulation by PKC would be much weaker in the case of $\mathrm{Ca}^{2+}$ oscillations of the bursting type. This would explain why, in contrast to simple periodic oscillations, bursting is insensitive to phorbol esters which activate PKC. No attempt was made in the present paper to incorporate regulation of the $\mathrm{IP}_{3}$ synthesis pathway by PKC. A mathematical model based on such regulation was previously proposed for $\mathrm{Ca}^{2+}$ oscillations, but only simple periodic oscillations were obtained in that model [10].

Bursting in our models occurs in a rather large domain in parameter space. The value of $v_{0}$ can, for example, be varied by $\pm 25 \%$ without a qualitative change of the behaviour. Chaos, however, occurs in a much smaller region of parameter space. Indications in favour of the existence of chaotic behaviour 
in $\mathrm{Ca}^{2+}$ dynamics has recently been reported for hepatocytes and pancreatic acinar cells [58].

Recently, Shen and Larter [32] have obtained complex $\mathrm{Ca}^{2+}$ oscillations and chaos in a model in which CICR is coupled with $\mathrm{Ca}^{2+}$-activated production of $\mathrm{IP}_{3}$. The latter mechanism was proposed by Meyer and Stryer [7] as a source for simple periodic oscillations, and has received recent experimental support [59]. The model analyzed by Shen and Larter [32] is closely related to the second model analyzed in the present work which is based on another type of regulation of the $\mathrm{IP}_{3}$ level which has received at least as much experimental support (see, e.g. [36]). Here, indeed, it is $\mathrm{IP}_{3}$ degradation which is activated by cytosolic $\mathrm{Ca}^{2+}$, rather than $\mathrm{IP}_{3}$ synthesis. As a result, the two models differ by their bursting pattern. While in the model of Shen and Larter , bursting is made up of a group of $\mathrm{Ca}^{2+}$ spikes that always return to the basal level, in our model (see Fig. 8), bursting consists of small-amplitude oscillations on a plateau phase separated by quiescent periods. The latter bursting resembles the complex oscillatory behaviour that can be observed in hepatocytes (see Fig. 1).

Both in our model and in the model studied by Shen and Larter [32] complex $\mathrm{Ca}^{2+}$ oscillations are autonomous as they occur in the absence of periodic forcing. Chaos and bursting in $\mathrm{Ca}^{2+}$ dynamics have also been obtained in a model for simple periodic oscillations driven by a periodic input [33]. In a four-variable model [33] $\mathrm{Ca}^{2+}$ oscillations of the bursting type have also been obtained in the absence of periodic forcing. This complex two-pool model incorporates four regulations that can have a destabilizing effect. These regulations are: $\mathrm{CICR}, \mathrm{Ca}^{2+}$-induced inactivation of the $\mathrm{IP}_{3}$-insensitive channels, $\mathrm{PKC}$-induced inactivation of the $\mathrm{IP}_{3}$-synthesis pathway and stimulation of phospholipase C (PLC) activity by both $\mathrm{IP}_{3}$ and $\mathrm{Ca}^{2+}$. The present study demonstrates that such a complex network of regulations is not required in order to generate bursting-type oscillations resembling those observed in experiments.

Taken together, the present results and those of others (see for example $[32,33]$ ) indicate that complex $\mathrm{Ca}^{2+}$ oscillations should not be uncommon, and that the phenomenon is even a likely one given the multiplicity of regulatory processes controlling $\mathrm{Ca}^{2+}$ signalling within the cell.

\section{Acknowledgements}

This work was supported by the programme Actions de Recherche Concertée (ARC 94-99/180) launched by the Division of Scientific Research, Ministry of Science and Education, French Community of Belgium, and by grant 3.4588 .93 from the Fonds de la Recherche Scientifique Médicale. G.D. is Chargé de Recherches du Fonds National de la Recherche Scientifique (Belgium).

\section{References}

[1] R.W. Tsien, R.Y. Tsien, Annu. Rev. Cell Biol. 6 (1990) 715.

[2] Y. Tsunoda, New Biologist 3 (1991) 3.

[3] M.J. Berridge, Nature 361 (1993) 315.

[4] C. Fewtrell, Annu. Rev. Physiol. 55 (1993) 427.

[5] M.J. Berridge, G. Dupont, Curr. Opin. Cell Biol. 6 (1994) 267.

[6] K. Kuba, S. Takeshita, J. Theor. Biol. 93 (1981) 1009.

[7] T. Meyer, L. Stryer, Proc. Natl. Acad. Sci. USA 85 (1988) 5051.

[8] A. Goldbeter, G. Dupont, M.J. Berridge, Proc. Natl. Acad. Sci. USA 87 (1990) 1461.

[9] S. Swillens, D. Mercan, Biochem. J. 271 (1990) 835.

[10] K.S.R. Cuthbertson, T.R. Chay, Cell Calcium 12 (1991) 97.

[11] G. Dupont, M.J. Berridge, A. Goldbeter, Cell Calcium 12 (1991) 73 .

[12] R. Somogyi, J.W. Stucki, J. Biol. Chem. 266 (1991) 11068.

[13] G. De Young, J. Keizer, Proc. Natl. Acad. Sci. USA 89 (1992) 9895.

[14] G. Dupont, A. Goldbeter, BioEssays 14 (1992) 485.

[15] G. Dupont, A. Goldbeter, Cell Calcium 14 (1993) 311.

[16] J. Keizer, G. De Young, J. Theor. Biol. 166 (1994) 431.

[17] Y.X. Li, J. Rinzel, J. Keizer, S.S. Stojilković, Proc. Natl. Acad. Sci. USA 91 (1994) 58.

[18] Y. Tang, H.G. Othmer, Biophys. J. 67 (1994) 2223.

「19] L. Sjödin, H.G. Dahlén, E. Gylfe, J. Physiol. 444 (1991) 763.

[20] R. Jacob, Cell Calcium 12 (1991) 127.

[21] T. Capiod, J. Noel, L. Combettes, M. Claret, Biochem. J. 275 (1991) 277.

[22] I. Marrero, A. Sanchez-Bueno, P.H. Cobbold, C.J. Dixon, Biochem. J. 300 (1994) 383.

[23] A.K. Green, C.J. Dixon, A.G. McLennan, P.H. Cobbold, M.J. Fisher, FEBS 322 (1993) 197.

[24] C.J. Dixon, P.H. Cobbold, A.K. Green, Biochem. J. 293 (1993) 757.

[25] C.J. Dixon, P.H. Cobbold, A.K. Green, Biochem. J. 309 (1995) 145.

[26] A.K. Green, P.H. Cobbold, C.J. Dixon, Biochem. J. 302 (1994) 949.

[27] I. Bezprozvanny, J. Watras, B.E. Ehrlich, Nature 351 (1991) 751 
[28] A. Fabiato, J. Gen. Physiol. 85 (1985) 247.

[29] K.A. Stauderman, M.M. Murawsky, J. Biol. Chem. 266 (1991) 19150.

[30] A. Atri, J. Amundson, D. Clapham, J. Sneyd, Biophys. J. 65 (1993) 1727.

[31] Y. Tang, J.L. Stepenson, H.G. Othmer, Biophys. J. 70 (1996) 246.

[32] P. Shen, R. Larter, Cell Calcium 17 (1995) 225.

「33] T.R. Chay, Y.S. Fan. Y.S. Lee. Int. J. Bifurcation Chaos 5 (1995) 595

[34] E.A. Finch, T.J. Turner, S.M. Goldin, Science 252 (1991) 443.

[35] C.W. Taylor, I.C.B. Marshall, TIBS 17 (1992) 403.

[36] K. Takazawa, M. Lemos, A. Delvaux, C. Lejeune, J.E. Dumont, C. Ermeux, Biochem. J. 268 (1990) 213.

[37] A. Goldbeter, O. Decroly, Y.X. Li, J.L. Martiel, F. Moran, Biophys. Chem. 29 (1988) 211.

[38] J. Rinzel, in: E. Teramoto and M. Yamaguti (Eds.), Mathematical Topics in Population Biology, Morphogenesis and Neurosciences, vol. 71, Lecture Notes in Biomathematics, Springer, Berlin, 1987, p. 267.

[39] A. Goldbeter, Biochemical Oscillations and Cellular Rhythms. The Molecular Bases of Periodic and Chaotic Behaviour. Cambridge University Press, London, 1996.

[40] I. Bezprozvanny, B.E. Ehrlich, Neuron 10 (1993) 1175.

[41] M. Iino, J. Gen. Physiol, 95 (1990) 1103.

[42] I. Parker, I. Ivorra, Proc. Natl. Acad. Sci. USA 87 (1990) 260.

[43] A.V. Zholus, S. Konnori, H. Ohashi, T.B. Boltun, J. Physiol. 481 (1994) 97.

[44] A. Fabiato, in: C.B. Frank, P. Bianchi, H. Keurs (Eds.), Excitation-contraction Coupling in Skeletal, Cardiac, and Smooth Muscle, Plenum Press, New York, 1992, p. 245.
[45] J. Schrenzel, N. Demaurex, M. Foti, C. Van Delden, J. Jacquet, G. Mayr, D.P. Lew, K.H. Krause, Biophys. J. 69 (1995) 2378.

[46] L.A. Segel, A. Goldbeter, P.N. Devreotes, B.E. Knox, J. Theor. Biol. 120 (1986) 151.

[47] M. Wakui, B.V.L. Potter, O.H. Petersen, Nature 339 (1989) 317.

[48] T. Meyer, D. Holowka, L. Stryer, Science 240 (1988) 653.

[49] P.S. Liu, Y.J. Lin, L.S. Kao, J. Neurochem. 56 (1991) 172.

[50] I.M. Robinson, R.D. Burgoyne, J. Neurochem. 56 (1991) 1587.

[51] K.A. Stauderman, R.A. McKinney, M. M Murawsky, Biochem. J. 278 (1991) 643.

[52] G.J. Law, J.A. Pachter, O. Thastrup, M.R. Hanley, P.S. Dannies, Biochem. J. 267 (1990) 359.

[53] P.D. Walton, J.A. Airey, J.L. Sutko, C.F. Beck, G.A. Mignery, T.C. Südhof, T.J. Deerinck, M.H. Ellisman, J. Cell Biol. 113 (1991) 1145.

[54] J.K. Foskett, D. Wong, J. Biol. Chem. 266 (1991) 14535.

[55] T. Matsumoto, H. Kanaide, Y. Shogakiuchi, M. Nakamura, J. Biol. Chem. 265 (1990) 5610.

[56] R. Bertram, M. Butte, T. Kienel, A. Sherman, Bull. Math. Biol. 57 (1995) 413.

[57] A. Goldbeter J.L. Martiel, in: H. Degn, A.V. Holden, L.F. Olser, (Eds.), Chaos in Biological Systems, Plenum Press, New York, 1987, p. 79.

[58] P. Strizhak, I. Magura, K. Yatsimirskii, A. Masyuk, J. Biol. Phys. 21 (1995) 233.

[59] M.D. Bootman, K.W. Young, J.M. Young, R.B. Moreton, M.J. Berridge, Biochem. J. 314 (1996) 347. 